\title{
Assessment of Potential Tooth Movement and Bite Changes With a Hard-Acrylic Sleep Appliance: A 2-Year Clinical Study
}

\author{
Nikola Vranjes, DDS ${ }^{1}$, Gene Santucci, DDS, MA², Karen A. Schulze, PhD, DDS², David Kuhns, $\mathrm{PhD}^{3}$, Allen Khai ${ }^{2}$ \\ ${ }^{1}$ The Snore Centre, Calgary, Alberta, Canada; ${ }^{2}$ University of Pacific, San Francisco, California; ${ }^{3}$ ProSomnus Sleep Technologies, Pleasanton, \\ California
}

\begin{abstract}
Study Objectives: The objective of this study was to test a proprietary hard-acrylic computer-aided design/computer-aided manufacturing $(\mathrm{CAD} / \mathrm{CAM})$ oral appliance with "retainer-like fit" and determine whether it resulted in tooth movement upon regular wear over a minimum 2-year period. Additionally, this study focused on determining patient acceptance of said device considering any reported side effects.

Methods: The 2-year clinical study reports on selected patients in whom sleep apnea has been diagnosed ( $\mathrm{n}=18)$ recruited from The Snore Center in Calgary. Patients were fitted with a ProSomnus® Sleep Appliance. Impressions were taken at baseline, after approximately 1 year, and after 2 years of use with the oral appliance. Models were marked, scanned, and scored; upper and lower anterior teeth crowding was assessed; and overjet and overbite were measured independently at the University of Pacific. Patients were surveyed on compliance, satisfaction with the appliance, and quality of life using the Sleep Apnea Quality of Life Index (SAQLI-10).

Results: This study shows a rigid OA made with proprietary CAD/CAM technology demonstrated no significant change in tooth position during the 2.3-year test period, or in bite changes per maximum intercuspation (MIP), as measured by overjet and overbite. At 2 years, the mean change in Little Irregularity Index for the lower anterior teeth was $0.007 \mathrm{~mm}(95 \%$ confidence interval $=[-0.03,0.05])$, which was not statistically different from zero $(\mathrm{P}>0.05)$. Patients were highly satisfied with the device and considered it beneficial. Conclusions: A key component to any treatment is patient's acceptance of the appliance and the willingness to wear the OA long term. When patient compliance is observed, hard-acrylic sleep appliances have little effect on tooth movement and MIP bite changes as shown by overjet/overbite.
\end{abstract}

Clinical Trial Registration: Health Research Ethics Board of Alberta: CTC-16-0108

Keywords: CAD/CAM technology, obstructive sleep apnea, oral appliance, tooth movement

Citation: Vranjes N, Santucci G, Schulze K, Kuhns D, Khai A. Assessment of potential tooth movement and bite changes with a hardacrylic sleep appliance: A 2-year clinical study. J Dent Sleep Med. 2019;6(2)

\section{INTRODUCTION}

Oral appliance therapy (OAT) has been shown to successfully treat patients with obstructive sleep apnea/hypopnea syndrome (OSAHS). ${ }^{1-3}$ However, OAT is often associated with the occurrence of side effects such as unwanted tooth movement. Positive airway pressure therapy is more commonly used to treat OSAHS, because of the reluctance to prescribe OAT as a result of these side effects. ${ }^{4}$ Even so, Pliska $^{5}$ does report on tooth movement due to tongue thrusting as a result of the use of positive airway pressure. Although OAT is an effective treatment for OSAHS along with its greater compliance, long-term use of some oral appliances has been shown to result in several side effects, including tooth movement. Past studies have shown this to result in significant effects, such as craniofacial changes, ${ }^{6-8}$ anteroposterior positioning of the molars, inclination of the upper and lower incisors, ${ }^{9}$ and irregularity of the lower incisors. ${ }^{10}$ According to the American Academy of Dental Sleep Medicine's published article on management of side effects, tooth movement (specifically occlusal changes represented by incisor changes, position of canines, decreased overjet/overbite, and altered occlusal contacts/bite changes) is one of the five significant concerns during the treatment of obstructive sleep apnea using an oral appliance. ${ }^{11}$ It was reported that there is a significant difference in anterior crowding using a flexible oral appliance without incisor coverage versus a rigid OA. ${ }^{10}$ The rigid OA demonstrated less tooth movement. Recently, new devices with proprietary computer-aided design/computer-aided manufacturing (CAD/CAM) technology have been introduced and made available to clinicians. This study aims to investigate the changes in tooth movement with a hard-acrylic rigid oral appliance, the ProSomnus ${ }^{\circledR}$ Sleep Appliance, over a 2-year period. This research project was based on an approved Internal Review Board (IRB) protocol. $^{12}$

\section{METHODS}

Patients were accepted into the study who were willing to return for follow-up impressions to be taken at the respective time points of 1 and 2 years. Patients were recruited from a pool of randomly selected patients $(n=53)$ from an earlier study ${ }^{3}$ with an acceptance criterion of body mass index less than $35 \mathrm{~kg} / \mathrm{m} 2$ and AHI greater than 10 events per hour. Eighteen of the 53 patients (14 male/4 
female) with sleep apnea were recruited for this study. Average patient age was 54 years, with an overall pretreatment AHI of 25.4 events per hour and posttreatment AHI of 6.7 events per hour (Table 1). Patients' pretreatment impressions were taken and the bite position captured using the George Gauge, and patients were fitted with a ProSomnus ${ }^{\circledR}$ Sleep Appliance (Figure 1) at The Snore Centre in Calgary. Patients were not fitted with a morning aligner. The patient's bite was determined by the MATRx (Zephyr Sleep Technologies, Calgary, AB, Canada) target position ${ }^{3}$ in which the bite position was at a percentage of maximum protrusion ranging from $40 \%$ to $100 \%$. Polyvinylsiloxane impressions were taken at baseline, after approximately 1 year (mean $=1.2$ years) and after 2 years (mean $=2.3$ years) of use with the oral appliance. Patients were surveyed on compliance and quality of life using the Sleep Apnea Quality of Life Index (SAQLI). The compliance survey asked patients to subjectively report on their compliance; patients were asked if they wore their appliance more than 4 hours per night, more than 5 days per week. The SAQLI survey was given to patients at the 1-and 2-year time points. The initial time point was unavailable as recruited patients had already been wearing their appliances upon study initiation. Models were marked, per the IRB, to scrub the patient data and to obscure the time points of each patient, from the measurement technician, to reduce measurement bias. Statistical analysis was completed by a separate party not involved in the measurement procedure. The mixedeffects model for analysis was chosen to provide the best estimates of the mean and confidence intervals. This model deals more adequately with the variable number of measurement replicates at each time point for each subject. Patient models (Figure 2) were scanned on a TRIOS lab scanner (3Shape, Copenhagen, Denmark) and scored using 3Shape OrthoAnalyzer software (3Shape, Copenhagen, Denmark). ${ }^{13}$ The upper and lower anterior teeth crowding was calculated using the Little Irregularity Index method ${ }^{14}$ (Figure 3). The models were placed in maximum intercuspation (MIP) and scanned, and overjet and overbite were measured using 3Shape OrthoAnalyzer software (Figure 4). Scanning and measurements were completed by the University of Pacific School of Dentistry.

\section{Table 1. Patient Profiles}

\begin{tabular}{|c|c|c|c|}
\hline Subjects & Avg age (years) & $\begin{array}{c}\text { Avg pre-AHI } \\
\text { (events/h) }\end{array}$ & $\begin{array}{c}\text { Avg post-AHI } \\
\text { (events/h) }\end{array}$ \\
\hline Male $(n=14)$ & 54 & 27.7 & 7.5 \\
\hline Female $(n=4)$ & 54.5 & 17.7 & 4.1 \\
\hline Overall $(n=18)$ & $54.1+/-10.0$ & $25.4+/-14.4$ & $6.7+/-7.5$ \\
\hline
\end{tabular}

Patients were given two surveys, the SAQLI-10 short version and one to measure compliance and satisfaction with the rigid oral appliance. The purpose of the surveys was to determine whether patients would wear the appliance, their satisfaction level, and whether the appliance was considered beneficial. The compliance metric will demonstrate that the tooth movement results are under the condition that patients wore the device.

\section{Statistical Analysis}

The mean change from baseline was estimated in a repeated-measures (mixed-effects) linear model. This model allows for missing values at certain time points and efficiently uses all the data to estimate effects. Time was treated as a categorical variable. The model also yields an estimate of the measurement error for an impression at a single time point.

\section{RESULTS}

At the beginning of the study, the anterior upper and lower arches were measured in each patient. The same measurements were done after an average of 1.2 years. Variation from time zero to year 1 for individual pairs of teeth, for example, between 27-26 on the lower arch and 89 on the upper arch, had an overall average shift of -.022 $\mathrm{mm}$ for the upper arch and $-.014 \mathrm{~mm}$ for the lower arch. The average difference for the total Little Irregularity Index for the same 18 patients at an average of 2.3 years for the upper arch was $-0.007 \mathrm{~mm}$. The average difference for the lower arch was $0.003 \mathrm{~mm}$ total. Within this study sample size, these differences were not shown to be statistically significant. The average change in the patients' overbite after 2.3 years was $0.0045 \mathrm{~mm}$ and for overjet $-.015 \mathrm{~mm}$.

\section{Lower Arch}

The mean change in Little Irregularity Index at 1 year was $-0.014 \mathrm{~mm}(95 \%$ confidence interval $[\mathrm{CI}]=-0.05$, $0.02)$, which was not statistically different from zero $(\mathrm{P}>0.05)$. The estimated measurement error was $0.05 \mathrm{~mm}$; the mean change is thus less than the measurement error. At 2 years, the mean change in Little Irregularity Index was $0.007 \mathrm{~mm}(95 \% \mathrm{CI}=-0.03,0.05)$, which was not statistically different from zero $(\mathrm{P}>0.05)$. The mixedeffects model estimates of the mean change in Little Irregularity Index are presented in Table 2 and the change in measures are presented graphically in Figure 5. The boxplot shows acceptable symmetry in the spread of the data for a sample set of this size. 
Figure 1. ProSomnus ${ }^{\circledR}$ Sleep Appliance.
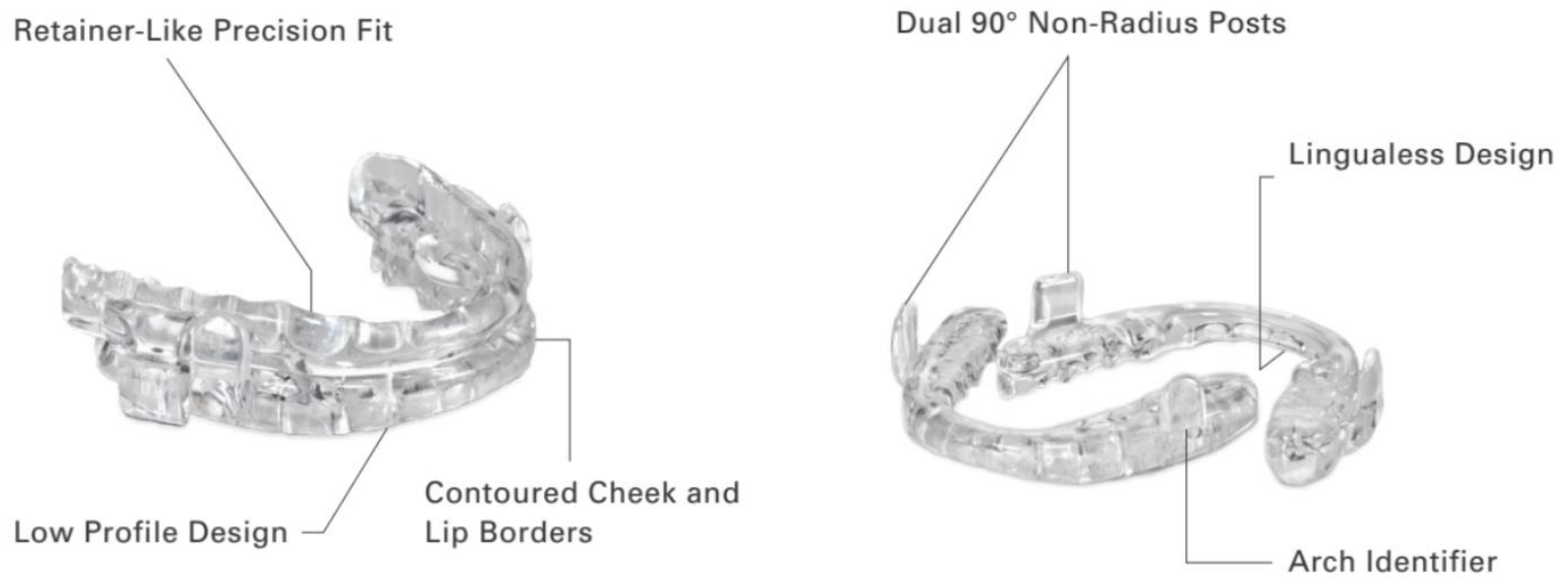

Figure 2. Examples of patient models taken at baseline, after 1 year and after 2 years, that visually demonstrate the low percentage of change in anterior tooth movement. $T=$ time, $Y R=$ year.



$\mathrm{T}=0$

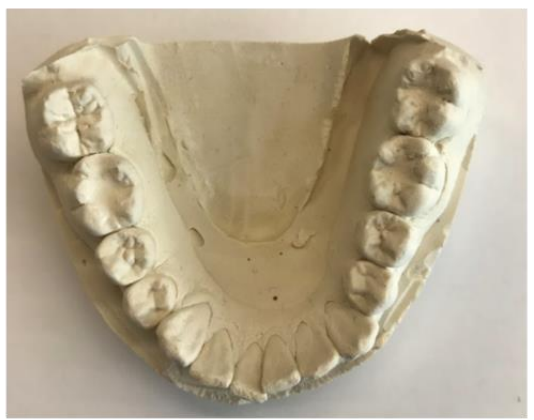

$\mathrm{T}=1 \mathrm{YR}$

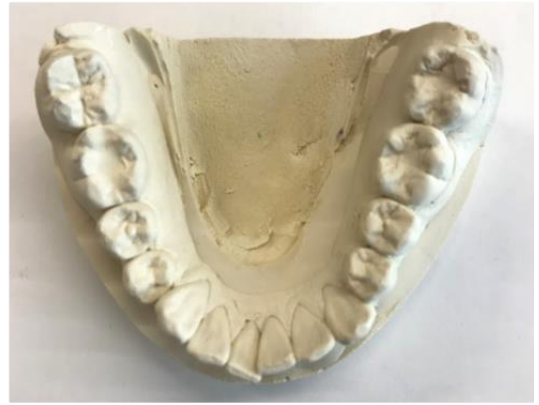

$\mathrm{T}=2 \mathrm{YR}$

Figure 3. Digitized patient models imported into the 3Shape OrthoAnalyzer module for measurements showing lower teeth crowding measured using the Little Irregularity Index.
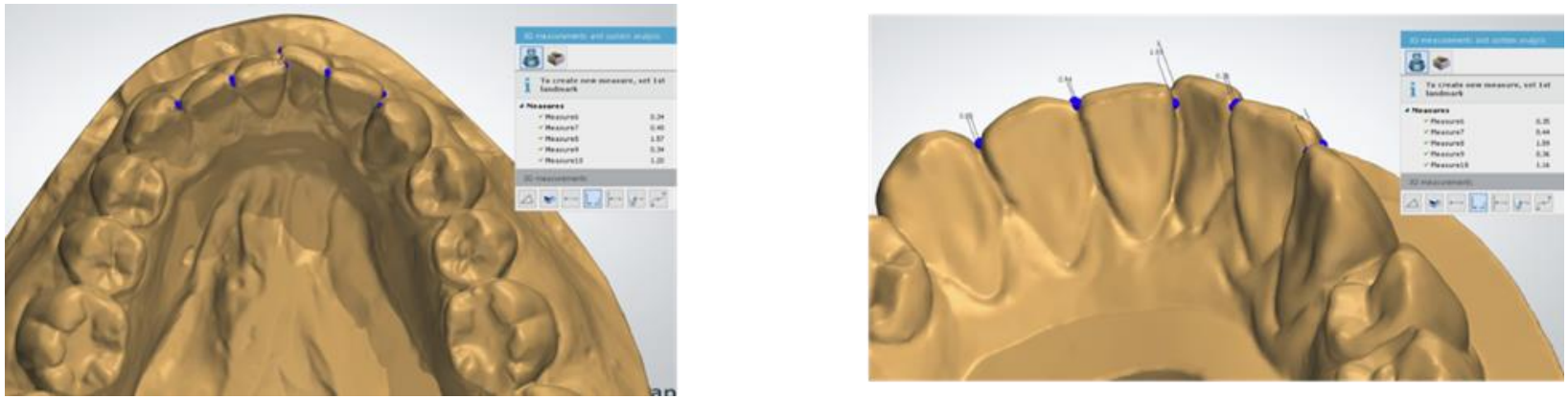
Figure 4. Hand-articulated models placed in maximum intercuspation scanned to measure overjet and overbite using 3Shape OrthoAnalyzer software.
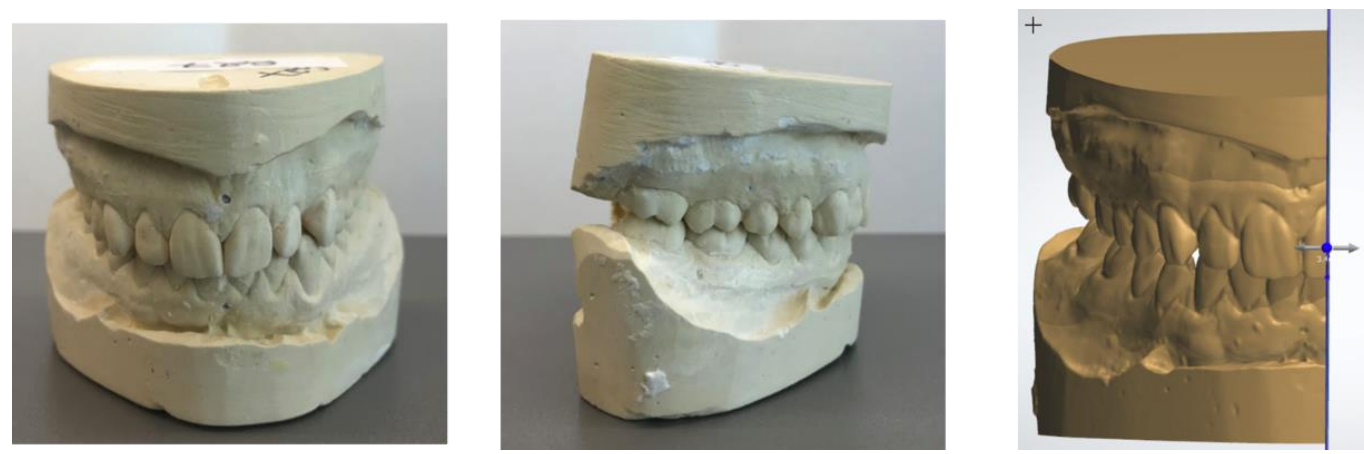

Figure 5. Lower arch and overjet analysis boxplots illustrating data symmetry.
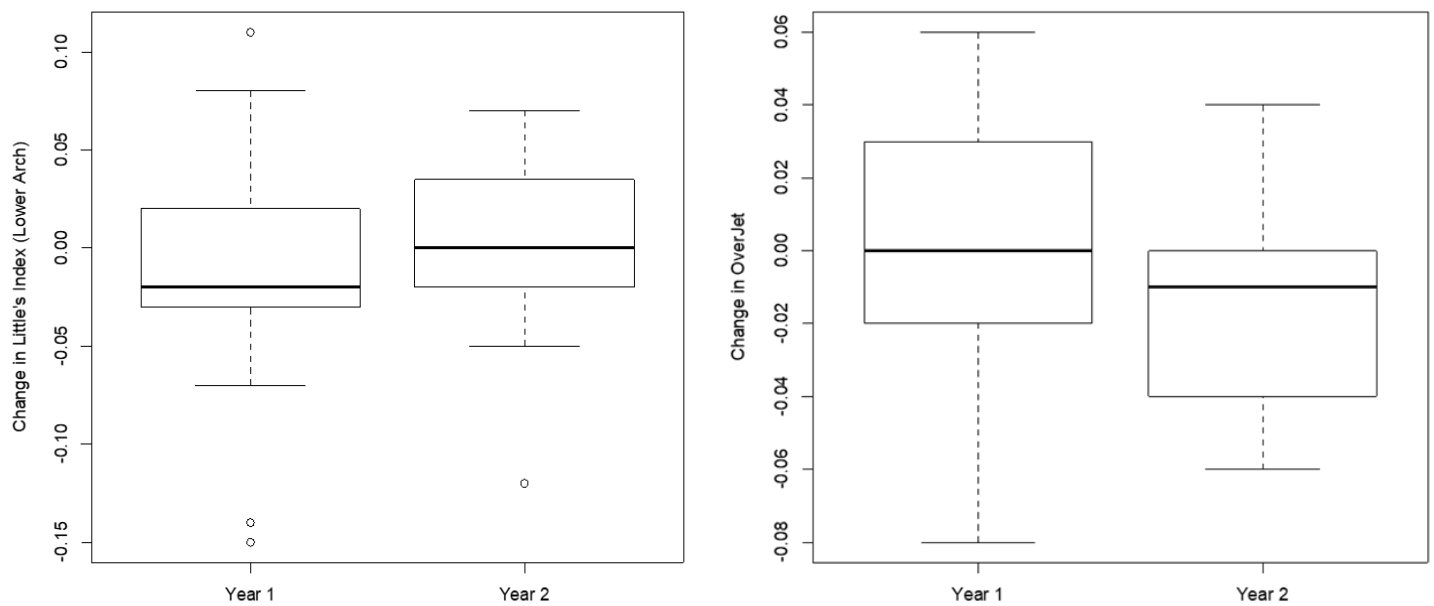

Table 2. Study Results (Repeated-Measures Model Least Squares Means)

\begin{tabular}{|l|l|l|l|l|}
\hline & \multicolumn{2}{|c|}{1.2 years } & \multicolumn{2}{c|}{2.3 years } \\
\hline & Mean change & $\begin{array}{l}95 \% \text { confidence } \\
\text { interval for mean } \\
\text { change }\end{array}$ & Mean change & $\begin{array}{l}95 \% \text { confidence } \\
\text { interval for mean } \\
\text { change }\end{array}$ \\
\hline Little Irregularity Index upper $(\mathrm{mm})$ & -0.022 & $-0.09,0.05$ & -0.002 & $-0.09,0.09$ \\
\hline Little Irregularity Index lower $(\mathrm{mm})$ & -0.014 & $-0.05,0.02$ & 0.007 & $-0.03,0.05$ \\
\hline Overjet (mm) & -0.002 & $-0.02,0.01$ & -0.008 & $-0.03,0.01$ \\
\hline Overbite (mm) & 0.003 & $-0.02,0.03$ & 0.0007 & $-0.03,0.03$ \\
\hline Intercanine distance upper $(\mathrm{mm})$ & 0.00001 & $-0.01,0.010$ & -0.01 & $-0.02,-0.0002$ \\
\hline Intercanine distance lower $(\mathrm{mm})$ & -0.003 & $-0.010,0.005$ & 0.004 & $-0.005,0.01$ \\
\hline
\end{tabular}




\section{Upper Arch}

The mean change in Little Irregularity Index at 1 year was $-0.022 \mathrm{~mm}(95 \% \mathrm{CI}=-0.09,0.05)$, which was not statistically different from zero $(\mathrm{P}>0.05)$. The estimated measurement error was $0.11 \mathrm{~mm}$; thus, the mean change was less than the measurement error. At 2 years, the mean change in Little Irregularity Index was $-0.002 \mathrm{~mm}(95 \% \mathrm{CI}$ $=-0.09,0.09)$, which was not statistically different from zero $(\mathrm{P}>0.05)$. The mixed-effects model estimates of the mean change in Little Irregularity Index are presented in Table 2.

\section{Overjet}

The mean change in overjet at 1 year was $-0.002 \mathrm{~mm}$ $(95 \% \mathrm{CI}=-0.02,0.01)$, which was not statistically different from zero $(\mathrm{P}>0.05)$. The estimated measurement error was $0.026 \mathrm{~mm}$; thus, the mean change was less than the measurement error. At 2 years, the mean change in overjet was $-0.008 \mathrm{~mm}(95 \% \mathrm{CI}=-0.03,0.01)$, which was not statistically different from zero $(\mathrm{P}>0.05)$. The mixedeffects model estimates of the mean in overjet are presented in Table 2 and the change measurements are presented graphically in Figure 5.

\section{Overbite}

The mean change in overbite at 1 year was $0.003 \mathrm{~mm}$ $(95 \% \mathrm{CI}=-0.02,0.03)$, which was not statistically different from zero $(\mathrm{P}>0.05)$. The estimated measurement error was $0.036 \mathrm{~mm}$; thus, the mean change was less than the measurement error. At 2 years, the mean change in overbite was $0.0007 \mathrm{~mm}(95 \% \mathrm{CI}=-0.03,0.03)$, which was not statistically different from zero $(\mathrm{P}>0.05)$. The mixedeffects model estimates of the mean change in overbite are presented in Table 2 .

\section{Upper Intercanine Distance}

The mean change in upper intercanine distance at 1 year was $0.00001 \mathrm{~mm}(95 \% \mathrm{CI}=-0.001,0.001)$, which was not statistically different from zero $(\mathrm{P}>0.05)$. The estimated measurement error was $0.014 \mathrm{~mm}$; thus, the mean change was less than the measurement error. At 2 years, the mean change in upper intercanine distance was $0.01 \mathrm{~mm}(95 \% \mathrm{CI}=-0.02,-0.0002)$, which was statistically different from zero $(\mathrm{P}<0.05)$. The mixed-effects model estimates of the mean change in upper intercanine distance are presented in Table 2.

\section{Lower Intercanine Distance}

The mean change in lower intercanine distance at 1 year was $-0.003 \mathrm{~mm}(95 \% \mathrm{CI}=-0.010,0.005)$, which was not statistically different from zero $(\mathrm{P}>0.05)$. The estimated measurement error was $0.011 \mathrm{~mm}$; thus, the mean change was less than the measurement error. At 2 years, the mean change in lower intercanine distance was $0.004 \mathrm{~mm}(95 \% \mathrm{CI}=-0.005,0.01)$, which was not statistically different from zero $(\mathrm{P}>0.05)$. The mixedeffects model estimates change in lower intercanine distance are presented in Table 2.

\section{SURVEY RESULTS}

Two surveys were given to patients to assess their acceptance of the device and the OAT, and to test for quality of life improvements (SAQLI). A total of $56 \%$ of patients $(n=10)$ responded to the device survey with an average duration of survey since appliance insertion of 2.3 $+/-0.4$ years. A total of $28 \%$ of patients $(n=5)$ completed two time points of the SAQLI survey. The device survey results are shown in Figure 6. Subjects were asked to rate their agreement on a 10-point scale, with 0 as "no agreement" and 10 as "full agreement." To determine patient compliance, patients were asked "Did you wear the device at least 5 days a week?" and "Did you wear the device at least 4 hours per night?". Based on the two questions, patients were $89.3 \%$ compliant in wearing the hard-acrylic sleep device from ProSomnus; furthermore, 7 out of 10 surveyed were $100 \%$ compliant per Medicare guidelines. Survey results showed high levels of positive feedback for all questions, with patients scoring on average $8.5 / 10$ points. While wearing the oral appliance, patients noted an improvement in sleep, the appliance was comfortable and easy to clean, they favored wearing the device for life, and will recommend the device to a friend.

The second survey given to patients during the study was the short version of the SAQLI. For this survey, five patients completed the surveys across two time points, year 1 and year 2, with an average duration of 10.4 months between surveys. For each survey question, the first data point was compared to the second data point, and if the answer was the same, the question scored a 0 . If there was improvement of one level, the score was a 1. If the answer decreased in favorability, the question was scored negative to the increments of change, e.g. $-1,-2-3$, etc. The most notable area of improvement in quality of life was the reduction in "irritation to snoring". However, even though on average the scores showed a positive 0.5 step improvement, statistically it would be difficult to make a strong conclusion. 


\section{Figure 6. Patient survey results $(10=$ most agreeable to the question)}

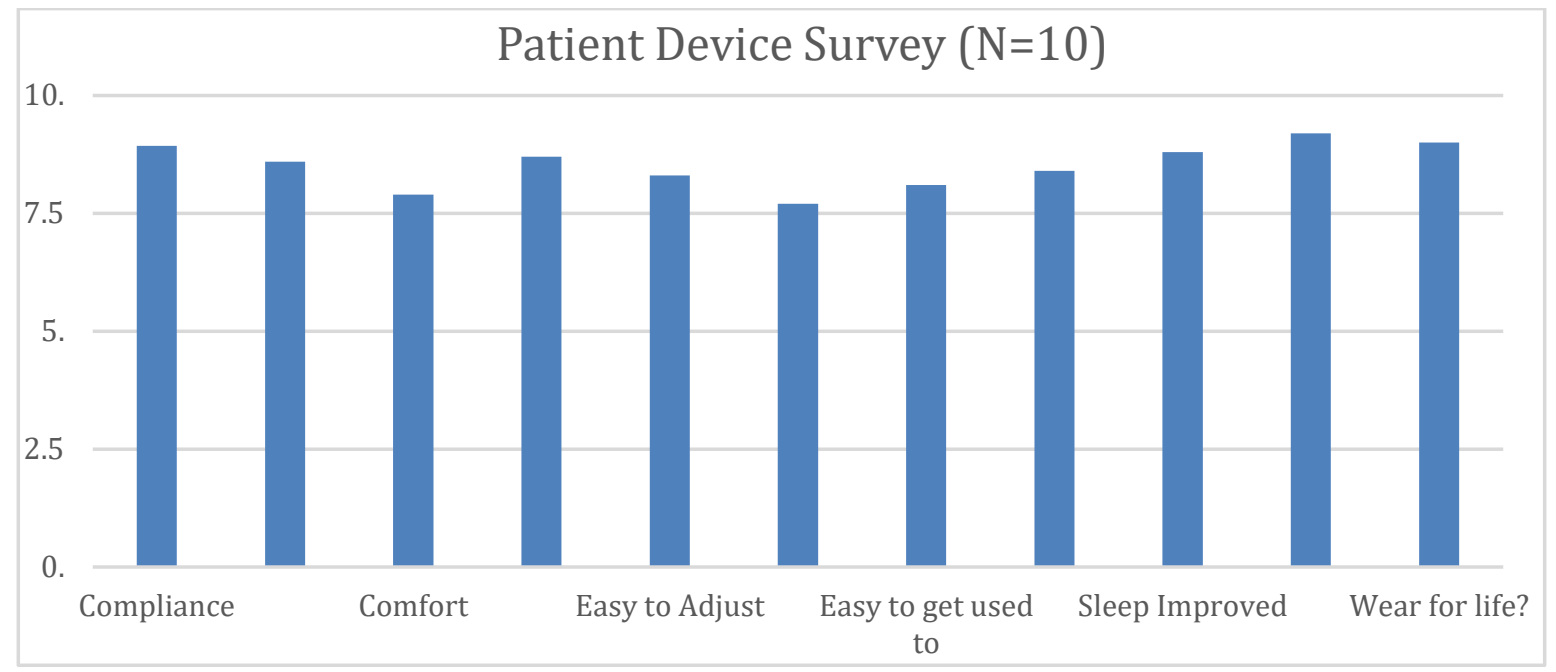

\section{DISCUSSION}

Sleep physicians and general dentists often cite side effects as a leading reason to avoid OAT. Of the many possible side effects related to OAT, tooth movement is one that is discussed, and is relatively easy to measure, especially with the advent of digital scanners. When it comes to choosing an oral appliance, clinicians now have a wide variety of appliance designs and materials. Appliances that have areas of stronger pressure, such as ball clasps or open areas, are much more likely to result in tooth movement, rather than an appliance that covers every tooth. ${ }^{11}$ Similarly, a hard acrylic rigid appliance is likely to provide more tooth stability than a softer appliance. ${ }^{10}$ An appliance designed with a proprietary $\mathrm{CAD} / \mathrm{CAM}$ process, made of hard acrylic with an even spacer around all areas in contact with the teeth, is therefore thought to provide enough stability to prevent tooth movement.

The study results show that when patients are compliant in wearing the appliance chosen in this study nightly (ProSomnus® Sleep Appliance) the data report minimal tooth movements, if any. Another significant finding discovered is that when models are handarticulated into MIP, overjet and overbite did not change. The changes noted in this study appear to be less than in previous oral appliance studies. ${ }^{10}$ Additionally, a key component to any treatment is acceptance of the oral appliance and the willingness to wear the device long term. Treatment compliance is required to obtain useful efficacy. The survey results demonstrated that patients wore the device at a high rate and would recommend the device to friends and family. This clinical study demonstrates that the proprietary CAD/CAM oral appliance minimizes tooth movement, and patients found the appliance comfortable and favorable. From a clinician's point of view, these study results can help alleviate concern from three audiences: the patient, a patient's referring dentist, and a sleep physician. These key findings provide additional evidence and information for dental/orthodontic practitioners when prescribing OAT for OSAHS.

The challenge of understanding the significance of tooth movement by an appliance is understanding the natural amount of tooth movement that may occur without an appliance. Future studies should include a control to establish what is typical tooth movement for a comparative patient population. This can be tricky as a treated versus untreated population may have different degrees of tooth movement. Because the patient may have many factors ${ }^{15}$ that could affect tooth mobility and subsequent tooth movement due to natural and iatrogenic sources, the authors chose a relative comparison of the patient's own data to specifically investigate the effect on the device to unwanted tooth movement. The study was limited by the ability to obtain surveys from all participants; many did not respond to further requests to create a complete dataset for the surveys. Understanding the change in the habitual bite was difficult in this case and the authors focused on the MIP of the patients' models with the data available. A more in-depth look at the patients' natural s bite via an intraoral scan, and cephalometric analysis and inspection of the temporal mandibular joint may provide a better understanding of bite changes. More studies are suggested to consider the many variables that contribute to occlusal changes and habitual bite changes and the tradeoffs to treating patients experiencing the effects of obstructive sleep apnea.

\section{ACKNOWLEDGMENTS}

An IRB protocol for the clinical trial in Canada was approved by the Health Research Ethics Board of Alberta, Canada.

The authors would like to thank and acknowledge support from University of Pacific, Arthur A. Dugoni School of Dentistry, Department of Preventive and 
Restorative Dentistry (San Francisco, CA). The authors also thank ProSomnus Sleep Technologies (Pleasanton, CA) for financial support, for technical support (3Shape OrthoAnalyzer Software) by Joshua Seefeldt at Argen (San Diego, CA), for statistical support from Colleen Kelly at Kelly Statistical Consulting (San Diego, CA), and the staff at Snore Centre Quarry Park (Calgary, AB, Canada).

\section{REFERENCES}

1. Hamoda MM, Kohzuka Y, Almeida FR. Oral appliance for the management of OS: An updated review of the literature. Chest. 2018;153(2):544-553.

2. Chan AS, Cistulli PA. Oral appliance treatment of obstructive sleep apnea: an update. Curr Opin Pulm Med. 2009;15(6):591596.

3. Remmers JE, Topor Z, Grosse J, et al. A feedback-controlled mandibular positioner identifies individuals with sleep apnea who will respond to oral appliance therapy. J Clin Sleep Med. 2017;13(7):871-880.

4. Ngiam J, Hons, Phil M, Cistulli PA. Think before sinking your teeth into oral appliance therapy. J Clin Sleep Med.2014;10(12):1293-1294.

5. Pliska BT, Almeida FR. Tooth movement associated with CPAP therapy. J Clin Sleep Med. 2018;14(4):701-702.

6. Uniken Venema J A.M., Stellingsma C, Doff MHJ, Hoekema A. Dental side effects of long-term obstructive sleep apnea therapy: A comparison of three therapeutic modalities. J Dent Sleep Med. 2018;5(2):39-46.

7. Doff MH, Hoekema A, Pruim GJ, Huddleston Slater JJ, Stegenga B. Long-term oral-appliance therapy in obstructive sleep apnea: a cephalometric study of craniofacial changes. $J$ Dent. 2010;38(12):1010-1018.

8. Tsuda H, Almeida F, Tsuda T, Moritsuchi Y, Lowe A. Craniofacial changes after 2 years of nasal continuous positive airway pressure use in patients with obstructive sleep apnea. Chest. 2010;138(4):870-874.

9. Rose JE, Staats R, Virchow C Jr, Irmtrud E. Occlusal and skeletal effects of an oral appliance in the treatment of obstructive sleep apnea. Chest.2002;122(3):871-877.

10. Norrhem N, Nemeczek H, Marklund M. Changes in lower incisor irregularity during treatment with oral sleep apnea appliances; Sleep Breath. 2017;21(3):607-613.

11. Sheats RD, Schell TG, Blanton AO, et al. Management of side effects of oral appliance therapy for sleep-disordered breathing. J Dent Sleep Med. 2017;4(4):111-125.

12. IRB Approval: Health Research Ethics Board of Alberta (https://hreba.ca/) HREBA.CTC-16-0108

13. Bukhari SAAB, Kaladhar A, Reddy C, et al. Evaluation of virtual models (3Shape OrthoSystem) in assessing accuracy and duration of model analyses based on the severity of crowding. The Saudi J Dent Res. 2017; 8 (1-2):11-18.

14. Little RM. The irregularity index: A quantitative score of mandibular anterior alignment. Am J Orthod. 1975;68(5):554563 .

15. Karthik R. Tooth Mobility - Causes and Treatment, December 28, 2018. http://www.drkarthikreddy.com/2015/12/28/toothmobility/ Accessed April 2, 2019.

\section{SUBMISSION \& CORRESPONDENCE INFORMATION}

\section{Submitted for publication February 15, 2019 \\ Submitted in final revised form March 19, 2019 \\ Accepted for publication March 29, 2019}

Address correspondence to: David Kuhns, PhD, ProSomnus Sleep Technologies, 5860 West Las Positas Blvd., Suite 25, Pleasanton, CA, 94588, Email: dkuhns@prosomnus.com

\section{DISCLOSURE STATEMENT}

Drs. Santucci and Schulze received no compensation outside of University of Pacific salaries for this article; Dr. Vranjes and staff were compensated for office time by ProSomnus Sleep Technologies; Mr. Khai was compensated for lab time by ProSomnus Sleep Technologies; Dr. Kuhns is VP of Technology and ProSomnus Sleep Technologies.

The authors Drs. Santucci, Schulze, Vranjes and Allen Khai have no conflicts of interest; Dr. David Kuhns is Chief Science Officer of ProSomnus Sleep Technologies. 